\title{
Intelligent Building Control System Based on Mobile Wireless Internet of Things
}

\author{
https://doi.org/10.3991/ijoe.v13i10.7746 \\ Geng Li, Yu-na Su( $\left.{ }^{\square}\right)$ \\ Henan Polytechnic University, Jiaozuo, China \\ suyuna@hpu.edu.cn
}

\begin{abstract}
In order to develop an advanced intelligent building control system, the new system based on mobile wireless Internet of things is designed. By analyzing the new technology, it is proved that the application and development of the Internet of things technology provides a new method for the effective control of intelligent buildings. In addition, a series of researches are carried out on the core technology of the construction equipment networking system. The hardware, software and wireless self-organization networks of the intelligent terminal are designed and implemented, and the key indexes of the intelligent terminal are tested. The fixed transmit power test results show that when the fixed emission frequency is $10 \mathrm{~dB}$, the communication distance is inversely proportional to the communication rate, which means that the smart terminal wireless network has strong ability to pass through obstacles. Based on the above finding, it is concluded that the stability of the mobile internet wireless network intelligent terminal can fully meet the performance requirements of intelligent building control, which lay is a good basis for its flexible use of the future.
\end{abstract}

Keywords - Intelligent building; Internet of things; wireless network; intelligent terminal

\section{Introduction}

With the increasing degree of economic and social informatization, the Internet of things and electronic information technology are gradually applied to the field of architecture. Therefore, it has become the focus of attention and discussion all over the world, which gave birth to the intelligent control system of intelligent building. According to relevant data, the energy consumption of construction electricity equipment can account for $80 \%$ of the total energy consumption of buildings, which has become the key point of restricting the sustainable development of the construction field [1]. As the number and types of electrical equipment in buildings are increasing, the traditional intelligent building control system cannot meet the needs of people's intelligent, diversified and convenient management and control. Therefore, an integrated control and management system for building electric equipment is urgently needed. 


\section{Literature review}

The Internet of things, as the name suggests, refers to the Internet between objects and objects". It was first proposed by Bill Gates, founder of the American Microsoft Corp, in the book "the road to the future". Japan, South Korea and the European Union and other countries have incorporated the Internet of things into the national development strategy, and invested heavily in the construction of Internet of things projects [2,3]. Salvadori F [4] uses wireless sensor digital systems to achieve realtime and efficient monitoring of factory power. Erol-Kantarci M [5] combines wireless technology with smart power and applies it to power monitoring system, which improves the effective utilization rate of home power. Wei Chuyuan designed [6] an intelligent building system for energy consumption monitoring and energy saving management by using the Internet of things technology. The application of the Internet of things of construction equipment makes the object intelligent, which can greatly reduce the energy consumption of buildings and electric power. At the same time, it can satisfy people's demand for convenient and intelligent buildings.

Intelligent building networking refers to the introduction of Internet of things technology into intelligent buildings under the premise of giving full consideration to the characteristics of the building itself. The wireless network and Ethernet are used to monitor the operation status and the external environment of the building electrical equipment in real time. The test data should be recorded, stored, shared and processed, so as to ensure the best performance of the building electrical equipment [7]. In this paper, the design and test of intelligent terminal is completed through the construction of the Internet of things system of building equipment, which provides the theoretical basis and technical support for centralized management and overall optimization of building equipment.

\section{$3 \quad$ Methods}

\subsection{System composition and architecture}

The definition of intelligent building networking is to integrate its own characteristics into architecture, and use the Internet of things technology in combination with Ethernet and wireless networks. After data fusion, real time information, such as parameter setting, operation condition and external environment, can be recorded, stored and shared through the network. It also provides sufficient data support for the optimization of intelligent building control system.

The intelligent building networking system is essentially a hybrid structure. It can be divided into the following sections, such as Server, data resource arrangement, control of network service and establishment of communication LAN. Intelligent terminal is the core component of the system. It completes the communication between upper layer server and lower layer device through wireless network and ethernet. Equipment refers to the basic equipment in the building, such as lights, air conditioning and so on. The induction device uses sensors to obtain personnel, environment 
conditions and other parameters in the building, and transmits them to the intelligent terminal and the server through the wireless network.

The specific structure of the intelligent building networking system: The intelligent building networking system is equipped with an intelligent terminal, which is responsible for building and maintaining the wireless communication network. The information obtained by the personnel and equipment can be transmitted to the intelligent terminal through the network. The intelligent terminal will unpack the packet and reorganize it according to the TCP / IP data frame format to send to the server. The communication server and the intelligent terminal adopt TCP/IP protocol to communicate with each other through ethernet. The intelligent terminal uploads data to the server through Ethernet and transfers it to the database for analysis, storage and sharing. Thus, the communication between the intelligent terminal and the server is completed. Web server extracts information from the database, providing users with policy formulation, command release, fault diagnosis and other services. However, users access the Web server via Wan, GPRS, 3G networks and other handheld devices, such as smartphones, to obtain the required information. This completes the control of building electrical equipment at any time and at any place.

\subsection{Hardware and software design of intelligent terminal}

Intelligent terminal is the core component of the construction equipment networking system. It sets up a bridge between the server and the equipment, and is responsible for the transmission, transformation and control of each data, information and parameter in the system. Therefore, the software and hardware design of smart terminals becomes critical. The correct operation of the software is based on good hardware, and the development of software determines the stability and security of the system. The design of hardware is related to the performance and quality of the whole system [8]. Based on the design of the hardware scheme, the hardware chip selection and circuit design are carried out. Then, the design of embedded software is completed, and the organization chart of intelligent terminal is shown as shown in figure 1.

\subsection{Design and implementation of wireless self-organization}

Compared with wired network, wireless network virtually increases its network transmission distance and position. The topology can enhance the efficiency of network control and maintenance, greatly reduce the security risk and improve the performance of all aspects of network [9]. The cluster network topology is composed of sub cluster nodes and the cluster head node that is responsible for the establishment and maintenance of the node data transmission mechanism. The cluster node will establish good communication within the agreement according to the fixed form and cluster head. On the basis of this, a topology of regional clustering network is obtained according to the characteristics of architecture itself. Intelligent terminal is the core of the structure, which is mainly responsible for the management of wireless network data, the establishment and maintenance of the system. Compared with other network topologies, the advantage of the topology of regional clustering networks lies 


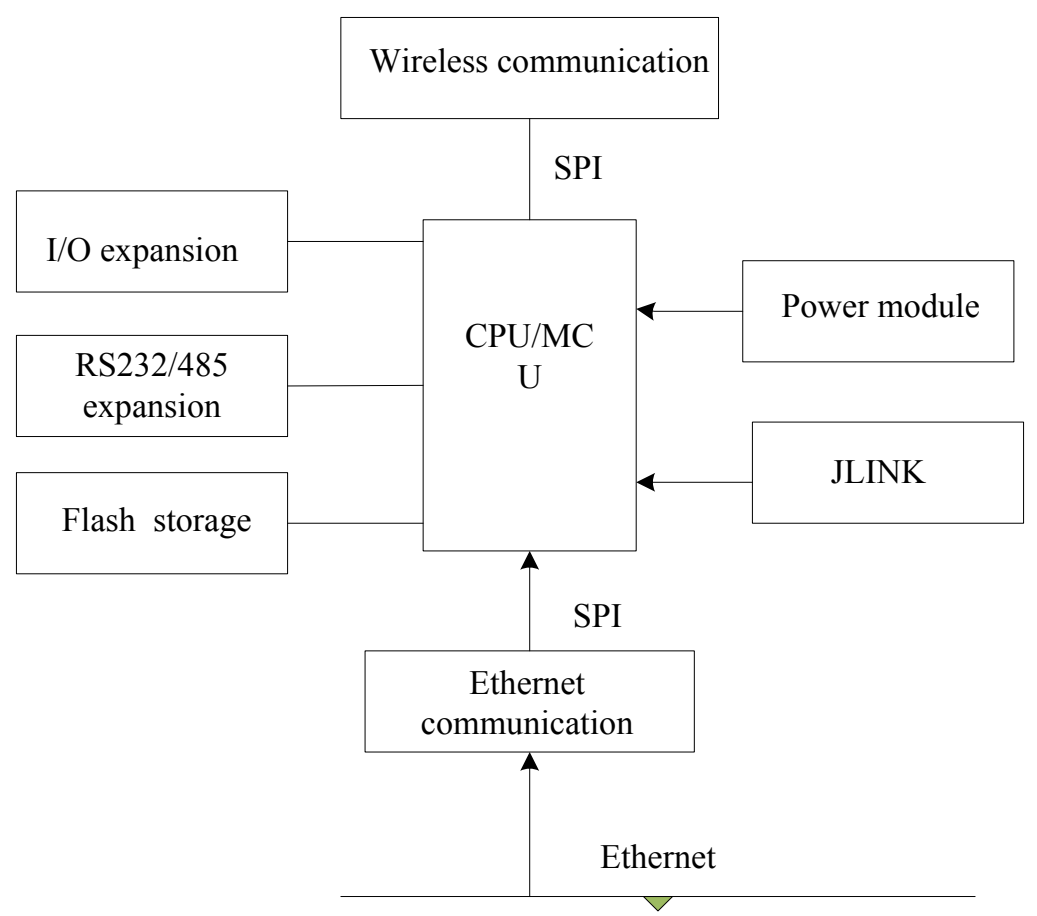

Fig. 1. Intelligent terminal organization structure

in the stability of backbone networks. This avoids the deformation of the network topology structure that leads to random variation of the sub cluster nodes, which greatly reduces the degree of influence and improves the reliability and safety of communication. Through the screening of wireless network topology, the design and implementation of intelligent terminal self-organization is completed [10]. Then, the basic communication performance, such as communication distance and packet loss rate, is tested. The three parallel tests are performed and the relevant data are recorded [11].

Communication distance: The $100 \mathrm{kbps}$ communication rate is selected in a room within the experimental building (the distance of the wall is $8 \mathrm{~m}$ ), and the transmission power of the intelligent terminal is changed. Different communication distances are obtained and the data is recorded [12]. On the contrary, the fixed transmit power is $10 \mathrm{~dB}$, and the communication speed is adjusted to obtain distance data and record it [13].

The packet loss rate in a certain intelligent terminal communication rate and transmit power, the equipment sends different size data packets to the intelligent terminal. Meanwhile, the number of terminals eventually received is counted and calculated in percentage terms

Networking test: The intelligent terminal and the underlying device are recorded on the network respectively, and the network is successfully established and the registration time is completed with the ms. 


\section{$4 \quad$ Results and discussion}

\subsection{Terminal hardware design results}

In order to get a well-run, safe and efficient intelligent building control system, the premise is to establish a stable and reliable hardware platform infrastructure. The intelligent terminal hardware based on the Internet of things takes STM32F103xx as its core microprocessor. CC1100 wireless transceiver, W5100 monolithic network communication chip, RS232 and RS485 peripheral extended interface, and a flash storage are used to complete the hardware design. The specific scheme is shown in figure 2 .

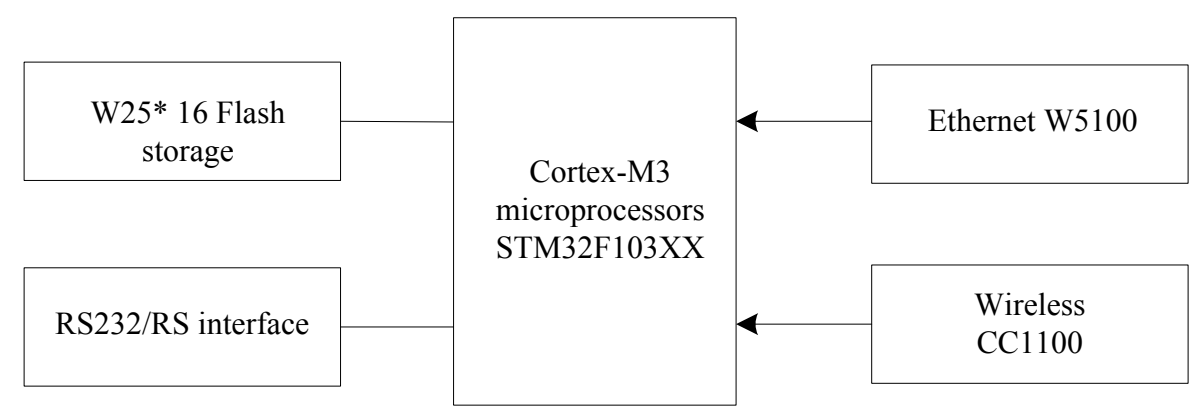

Fig. 2. Overall structure diagram of hardware design

\subsection{Software design result analysis}

Intelligent terminal software design is based on W5100 Ethernet communication program. It mainly includes the design and implementation of DHCP protocol and TCP/IP communication protocol. DHCP is the abbreviation of dynamic host configuration protocol, which means dynamic host configuration protocol. Its implementation is based on UDP protocol, server / client, and its dynamic acquisition process is shown in table 1. Intelligent terminal uses the DHCP protocol to realize the allocation of the network server / client address, and makes the IP address acquisition process more flexible and simple.

Table 1. Specific DHCP dynamic acquisition process

\begin{tabular}{|c|l|l|}
\hline No. & \multicolumn{1}{|c|}{ Process } & \multicolumn{1}{c|}{ Specific description } \\
\hline 1 & ICMP request, answer & It ensures that the terminal can access the network properly \\
\hline 2 & ARP request & It detects whether the acquired IP address is occupied \\
\hline 3 & ARP answer & When the terminal is accessed, it responds with its own IP \\
\hline 4 & UDP data transceiver & $\begin{array}{l}\text { The DHCP packet interaction is finished between the intelligent terminal } \\
\text { and the server }\end{array}$ \\
\hline 5 & DHCP protocol & The IP address dynamic acquisition process is finished \\
\hline
\end{tabular}


Transmission Control Protocol/Internet Protocol (TCP/IP) is a hierarchical structure. The upper layer is the transmission control protocol used to complete the information unpacking process. The lower layer is the Internet communication protocol used to properly manage the packet address. Its structural model is shown in figure 3. Based on the establishment of the underlying data communication, the application layer is used to complete the operation of the user program. Transport layer is the guarantee of the reliability of data transmission, mainly responsible for establishing, maintaining and controlling the transmission link. The network layer is the transport layer service, which provides the transparent service of routing and error control to satisfy the user's demand. As the bottom layer protocol, the link layer provides the support services for the network layer.

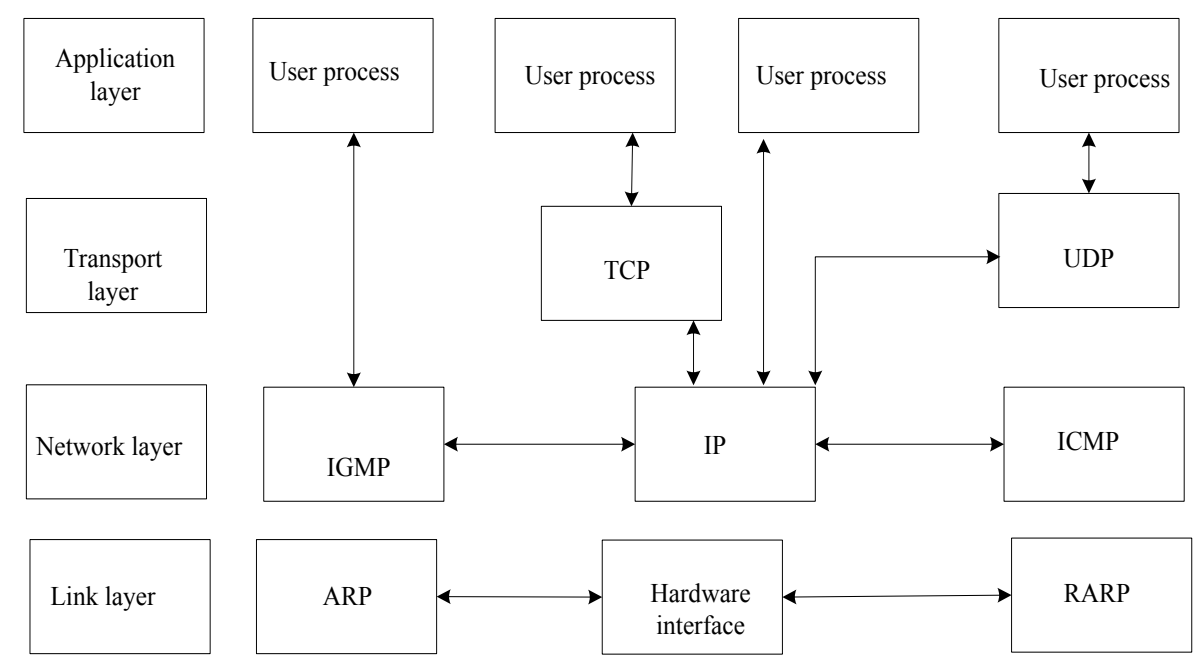

Fig. 3. TCP / IP protocol structure model

\subsection{Performance analysis of intelligent building system test}

Communication distance test. In the case of intelligent terminal node with different transmitting power and different communication rate, the communication distance of wireless network is measured, and the results are shown in figure 4 and figure 5.

Fig. 4 shows that the communication distance increases with the increase of transmit power at fixed $100 \mathrm{kbps}$ communication rate. In the same case of the obstacle, the transmission distance becomes far away when the transmitting power increases. This shows that the smart terminal wireless network has strong ability to pass through obstacles. As shown in figure 5, when the fixed emission frequency is $10 \mathrm{~dB}$, the communication distance is inversely proportional to the communication rate. Moreover, as communications speed decreases, the ability of wireless networks to move across the same barrier increases. 


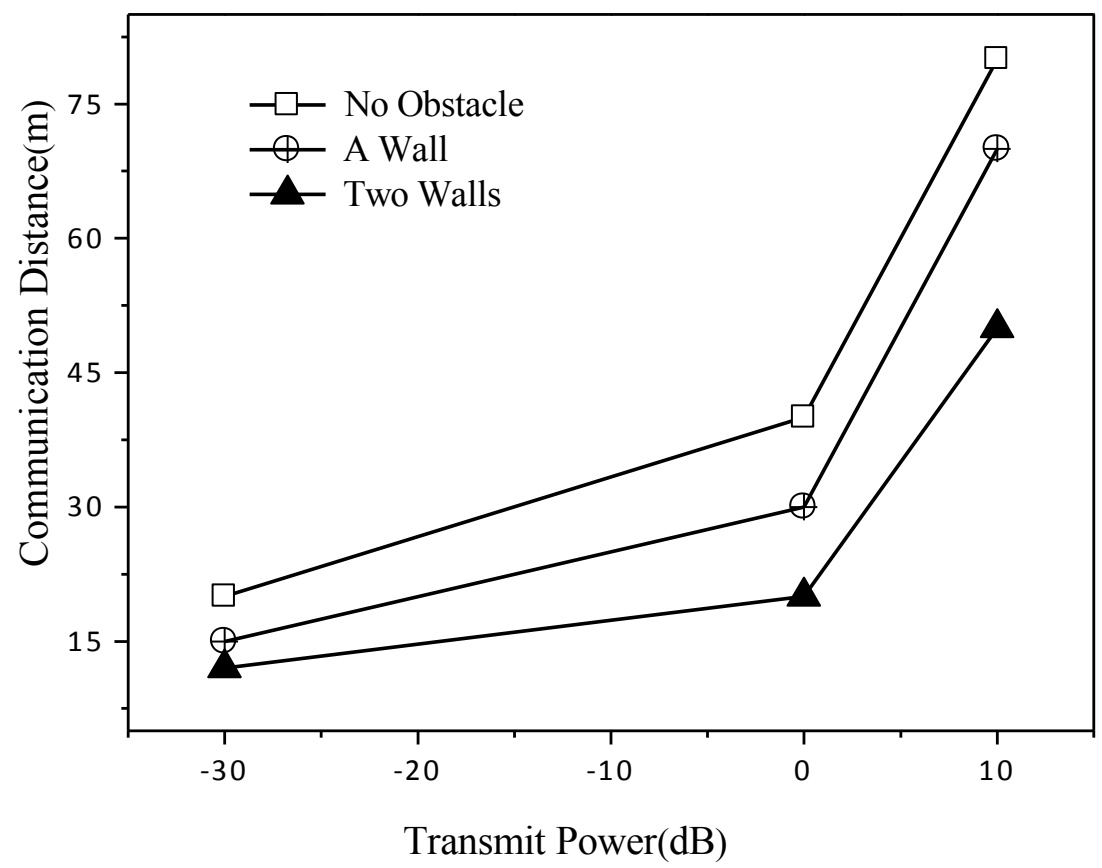

Fig. 4. Fixed communication rate test results

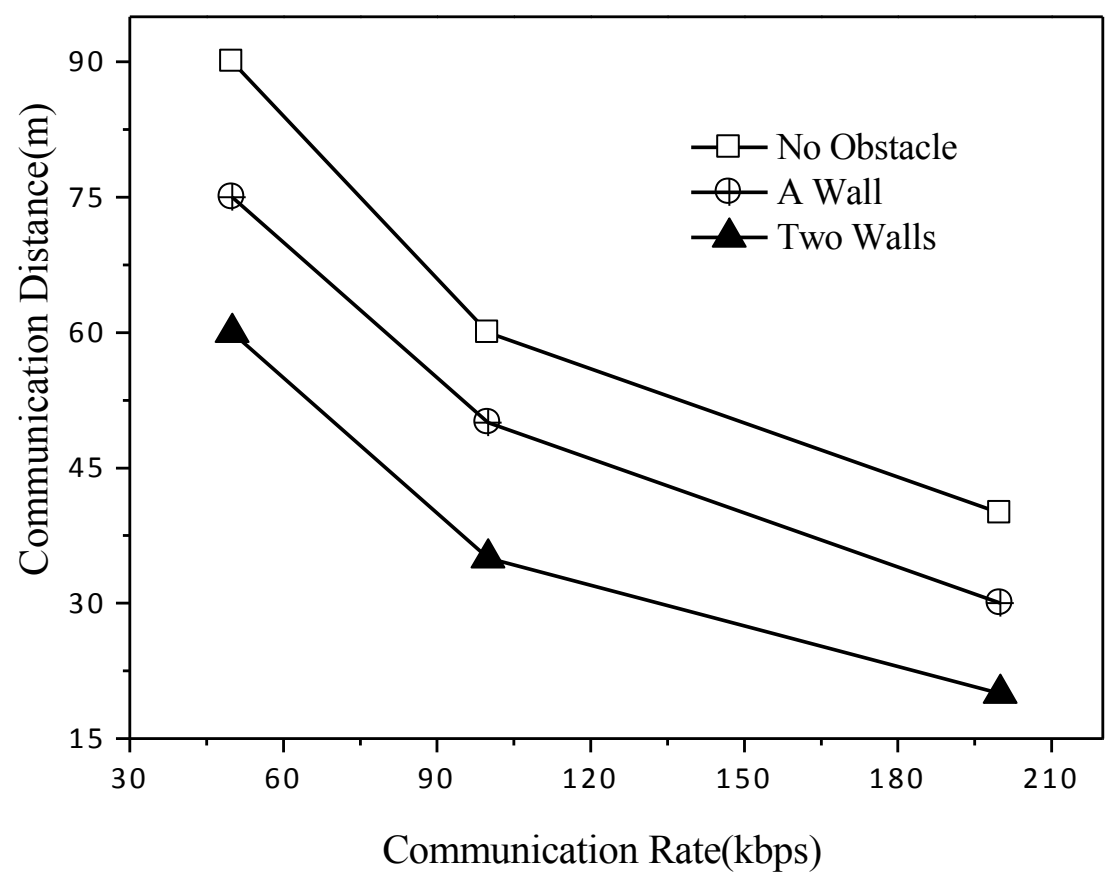

Fig. 5. Fixed transmit power test results 


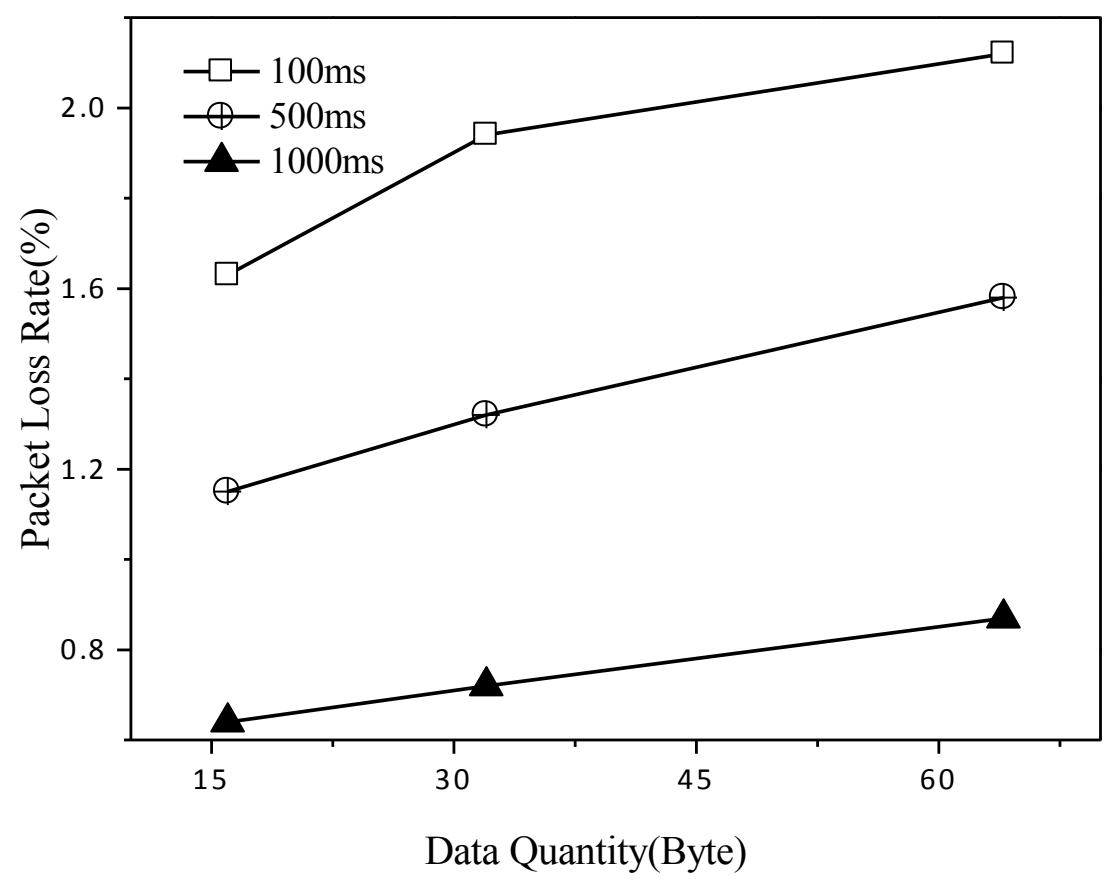

Fig. 6. Packet loss rate test results

Packet loss rate test. Under certain conditions of transmitting power and communication speed, the number of packets received by intelligent terminal in a given period of time is calculated. The results are shown in figure 6. As can be seen from the diagram, the size of the data and the time interval of the data transmission will affect the packet loss rate. The amount of data is small, the transmission interval will become longer, and the packet loss rate will decrease correspondingly.

Networking test. In a barrier free room, the smart terminal and the underlying device are powered. The time needed for the terminal to set up the network, the equipment to join the network, and the registration network are recorded separately. Test results are shown in table 2.

Table 2. Networking test results

\begin{tabular}{|l|c|}
\hline \multicolumn{1}{|c|}{ Test project } & Time (ms) \\
\hline Intelligent terminal networking time & 875 \\
\hline Bottom equipment netting time & 634 \\
\hline Device complete registration time & 458 \\
\hline
\end{tabular}




\section{Conclusion}

Combined with the Internet of things technology and intelligent building control system, on the basis of in-depth study of intelligent terminal, the human centered building equipment networking system was established. First of all, the whole scheme of intelligent terminal of building control system based on Internet of things is designed. At the same time, STM32F103xx microprocessor is used to design CPU core hardware circuit. At the same time, CC1100 chip and W5100 Ethernet chip are used to complete the hardware design. On this basis, the software design of intelligent terminal DHCP protocol and TCP/IP protocol communication is completed. Finally, the intelligent terminal, communication distance, packet loss rate and other performance tests are carried out. The results show that the construction of intelligent building control system has realized the basic goal of building energy conservation based on the Internet of things. Intelligent terminal of building equipment and Internet of things system has good testing performance. It can deal with all kinds of complex communication environment in the building and realize the wireless communication between server and device in real sense.

To sum up, it is concluded that the Internet of things has achieved good results in construction related fields. In order to cope with the risks that may arise during the future development, it is necessary to further optimize and upgrade the hardware and software of the system. Some comprehensive and sophisticated assessments and tests should be carried out for the index, security and stability of intelligent terminal. Therefore, the intelligent building control system based on mobile internet wireless Internet of things has higher stability, stronger application and wider application. The momentum of future development should not be underestimated.

\section{References}

[1] Ryu, C. S. (2015). Iot-based intelligent for fire emergency response systems. International Journal of Smart Home, 9(3), 161-168. https://doi.org/10.14257/ijsh.2015.9.3.15

[2] Mamun, M. A. A., Hannan, M. A., Hussain, A., \& Basri, H. (2016). Theoretical model and implementation of a real time intelligent bin status monitoring system using rule based decision algorithms. Expert Systems with Applications, 48(C), 76-88. https://doi.org/10.1016/j.eswa.2015.11.025

[3] Park, S., Park, S., Byun, J., Yu, Y., \& Park, S. (2015). Design of building energy autonomous control system with the intelligent object energy chain mechanism based on energyiot. International Journal of Distributed Sensor Networks,2015,(2015-11-1), 2015, 1-9. https://doi.org/10.1155/2015/931792.

[4] Atam, E., \& Helsen, L. (2016). Control-oriented thermal modeling of multizone buildings: methods and issues: intelligent control of a building system. IEEE Control Systems, 36(3), 86-111. https://doi.org/10.1109/MCS.2016.2535913

[5] Manic, M., Wijayasekara, D., Amarasinghe, K., \& Rodriguez-Andina, J. J. (2016). Building energy management systems: the age of intelligent and adaptive buildings. IEEE Industrial Electronics Magazine, 10(1), 25-39. https://doi.org/10.1109/MIE.2015.2513749 
[6] Zorzi, M., Gluhak, A., Lange, S., \& Bassi, A. (2010). From today's intranet of things to a future internet of things: a wireless- and mobility-related view. IEEE Wireless Communications, 17(6), 44-51. https://doi.org/10.1109/MWC.2010.5675777

[7] Piyare, R., \& Lee, S. R. (2013). Towards internet of things (iots):integration of wireless sensor network to cloud services for data collection and sharing. International Journal of Computer Networks \& Communications, 5(5), 59-72. https://doi.org/10.5121/ijcnc.2013.5 505

[8] Gubbi, J., Buyya, R., Marusic, S., \& Palaniswami, M. (2012). Internet of things (iot): a vision, architectural elements, and future directions. Future Generation Computer Systems, 29(7), 1645-1660. https://doi.org/10.1016/j.future.2013.01.010

[9] Abbas, Z., \& Yoon, W. (2015). A survey on energy conserving mechanisms for the internet of things: wireless networking aspects. Sensors, 15(10), 24818-24847. https://doi.org/10.3390/s151024818

[10] Dhillon, H. S., Huang, H., \& Viswanathan, H. (2015). Wide-area wireless communication challenges for the internet of things. IEEE Communications Magazine, 55(2), 168-174. https://doi.org/10.1109/MCOM.2017.1500269CM

[11] Chen, D., Chang, G., Sun, D., Jia, J., \& Wang, X. (2012). Lightweight key management scheme to enhance the security of internet of things. International Journal of Wireless \& Mobile Computing, 5(2), 191-198. https://doi.org/10.1504/IJWMC.2012.046773

[12] Banerjee, D., Dong, B., Taghizadeh, M., \& Biswas, S. (2014). Privacy-preserving channel access for internet of things. IEEE Internet of Things Journal, 1(5), 430-445. https://doi.org/10.1109/JIOT.2014.2346513

[13] Daokun Ma, Qisheng Ding, Zhenbo Li, Daoliang Li, \& Yaoguang Wei. (2012). Prototype of an aquacultural information system based on internet of things e-nose. Intelligent Automation \& Soft Computing, 18(5), 569-579. https://doi.org/10.1080/10798587.2012.1064 3266

\section{$7 \quad$ Authors}

Geng Li and Yu-na Su are with School of Physics \&Electronic Information Engineering, Henan Polytechnic University, Jiaozuo 454003, China.

Article submitted 04 September 2017. Published as resubmitted by the authors 20 October 2017. 